\title{
Characterization of Laser Produced Tetrakis (Dimethylamino) Ethylene Plasma in a High-Pressure Background Gas
}

\author{
Kamran Akhtar, Member, IEEE, John E. Scharer, Senior Member, IEEE, Shane Matthew Tysk, and \\ C. Mark Denning, Member, IEEE
}

\begin{abstract}
We present an interferometric and spectroscopic characterization of ultraviolet (UV) laser photoionization of a low ionization potential organic vapor, tetrakis (dimethylamino) ethylene (TMAE), seeded in high-pressure air component gases. These experiments are performed to explore the feasibility of using an electrodeless UV laser preionization of TMAE to initiate a plasma seeded in atmospheric pressure gases that can later be sustained by radiofrequency ( $R F)$ power by inductive wave coupling, thereby reducing the initiation RF power budget. A large volume $(500 \mathrm{cc})$, high-density $\left(\sim 10^{13} \mathrm{~cm}^{-3}\right)$, electrodeless plasma is created by single-photon, $193 \mathrm{~nm}$ excimer laser ionization. 105-GHz millimeter-wave interferometry along with optical spectroscopy is employed to investigate the plasma formation and decay characteristics. The TMAE plasma decay mechanisms including two-body and three-body recombination with and without high pressure gases are examined and the dominant loss processes discussed and evaluated. Both density and optical emission measurements show a delay of $140 \pm 10 \mathrm{~ns}$ in the peak plasma density and emission indicating that the dominant ionization process is delayed ionization via excitation of super-excited states. The experiment also shows that TMAE remains a viable seed gas for UV ionization in the presence of air for $t \leq 10 \mathrm{~min}$.
\end{abstract}

Index Terms-High-pressure plasmas, laser ionization and breakdown of plasmas, plasma emission.

\section{INTRODUCTION}

$\mathbf{T}$ HERE has been a great deal of recent effort to develop an improved method where large volumes $(\sim 1000-5000 \mathrm{cc})$ of atmospheric pressure plasmas at higher densities $\left(\sim 10^{13} \mathrm{~cm}^{-3}\right)$ can be initiated and sustained with a reduced power budget. These plasmas find applications in materials processing [1], biological decontamination [2] and modification of drag at supersonic speeds [3]. They can also provide protection from jamming and electronic countermeasures and modify the radar cross section and signature of aircraft [4], [5]. The theoretical minimum power density (power per unit volume: $\mathrm{P} / \mathrm{V}$ ) required to initiate and sustain an air plasma discharge, has been estimated for an equilibrium Maxwellian plasma for atmospheric pressures at sea level up to $300000 \mathrm{ft}$ [5], [6]. The model is based on an electron-beam delta function excitation and electric field sustainment. In order to maintain

Manuscript received October 30, 2003; revised January 13, 2004. This work was supported by Air Force Office of Scientific Research under Grant F49620-03-1-0252.

The authors are with the Department of Electrical and Computer Engineering, University of Wisconsin, Madison, WI 53706 USA (e-mail: kamran@ cae.wisc.edu).

Digital Object Identifier 10.1109/TPS.2004.826115 an air plasma density of $\sim 10^{13} \mathrm{~cm}^{-3}$ at sea level, the required minimum power density is $9 \mathrm{~kW} / \mathrm{cm}^{3}$. However, at higher altitudes of $30000 \mathrm{ft}$ to $60000 \mathrm{ft}$, with corresponding atmospheric pressures of 200-50 torr, the theoretical required power density is reduced to $2 \mathrm{~kW} / \mathrm{cm}^{3}$ and $660 \mathrm{~W} / \mathrm{cm}^{3}$, respectively.

The inductively coupled radiofrequency $(\mathrm{RF})$ power required to ionize a high-pressure air is much higher than the RF power $\left(\sim 9 \mathrm{~kW} / \mathrm{cm}^{3}\right)$ needed to sustain the plasma at sea level. In an atmospheric pressure plasma torch, a $300 \mathrm{kV}$ potential was required to initiate a discharge, whereas only $100 \mathrm{~V}$ was needed to maintain the discharge with operating currents of 200-600 A [7], [8]. However, once the seed discharge has been initiated by means such as UV flash lamp or laser, the plasma can be sustained at a much lower RF power level. A seed plasma can also be created by placing electrodes inside the chamber. The small plasma formed by the spark is localized between the electrodes. If the electrode is located close to the RF antenna so as to provide the required plasma load, arcing from the RF source to the electrode can occur. In addition, plasma bombardment of the electrode will result in deterioration over time. Therefore, we seek an electrodeless method for creating a large volume $(500 \mathrm{cc})$ seed plasma that will provide a good plasma load for efficient coupling via pulsed inductive sources. In addition, the long axial extent $(100 \mathrm{~cm})$ of the laser seed plasma can allow enhanced RF penetration and ionization well away from the $20-\mathrm{cm}$ axial extent of the antenna. The possibility of initiating a discharge by 193-nm laser photoionization of an organic gas tetrakis (dimethylamino) ethylene (TMAE) seeded in high-pressure background argon gas that was later sustained by inductive coupling of RF wave has been demonstrated by Kelly et al. [9]. This concept is further investigated to create a laser- initiated RF-sustained discharge with 760 torr air constituents as the background gas to determine the ionization mechanisms and the plasma decay processes of the seed plasma. Previous experiments by our group [9]-[12] have shown that a high initial density $\left(\sim 10^{13} \mathrm{~cm}^{-3}\right)$, long $(\sim 100 \mathrm{~cm})$ TMAE plasma can be efficiently created by a 193 -nm laser in argon and nitrogen. UV radiation from arrays of sparkboards have also been used to photoionize TMAE seeded in helium [13], [14]. However, to our knowledge, no previous detailed investigation of laser produced TMAE plasma formation and density decay processes has been carried out in the presence of air constituent gases.

In this regard, knowledge of the detailed decay characteristics of a laser-produced TMAE plasma after the application of a 20-ns laser pulse, in the presence of the background air 
constituent gases, is essential to determine the efficiency of subsequent inductive RF wave power coupling to sustain the discharge. The presence of air components such as oxygen at room temperature has a substantial impact on the plasma formation and plasma decay process through the electron attachment process [21]. In order to achieve efficient RF sustainment of a laser-preionized TMAE seed plasma, the following scientific issues have to be resolved: 1) The effect of the background gas on the formation and decay characteristics of the TMAE plasma; 2) the role of delayed ionization; 3) whether the lifetime of the laser-produced TMAE plasma is long enough such that RF power can be coupled efficiently through inductive wave coupling at lower power levels to sustain the plasma; and 4) the time scale for modification of TMAE vapor due to its chemical interaction with oxygen, which could reduce its viability as a readily UV ionized seed gas in air. In addition, the presence of background gas makes the plasma very collisional and, therefore, a plasma diagnostic that measures plasma collisionality and recombination losses is also required. Since our previous fast (10 ns) Langmuir probe (LP) measurements [10] could only be carried out $100 \mathrm{~ns}$ after the application of the laser pulse when the plasma is in a quiescent decay state, many physical processes, such as delayed ionization, present during the formation and early stages of the decay of the TMAE plasma could not be examined. Millimeter-wave (mm-wave) interferometry [15] along with the fast emission spectroscopy is used to obtain the full temporal characteristics of the TMAE plasma.

The goal of this research is to demonstrate the feasibility of laser preionization of TMAE plasma seeded in atmospheric air that could be efficiently sustained by reduced RF power. In order to accomplish this, we have examined nitrogen, oxygen and air as well as argon and helium background gases in an experiment that incorporates a large size plasma chamber $(5000 \mathrm{cc})$ and adequate vacuum conditions $\left(10^{-6}\right.$ torr base pressure). The temporal development of the plasma during and after the application of the $20 \mathrm{~ns}$ laser pulse, along with the axial average density $\left\langle n_{e}(t, z)\right\rangle_{d}$ profile (d is the plasma diameter), is presented. The experimental system and diagnostics are presented in Section II, experimental results are discussed in Section III, and a summary is presented in Section IV.

\section{EXPERIMENT}

\section{A. Laser Plasma System}

The general schematic of the laser-initiated and RF-sustained plasma experiment is shown in Fig. 1. In this paper we present only the laser ionization of TMAE seeded in high-pressure background gas. In these experiments the RF coil has not been energized but will be in future experiments. A uniform intensity ultraviolet (UV) beam of $193 \mathrm{~nm}$ wavelength is produced using an excimer laser (Lumonics Pulsemaster PM-842) that runs in the $\operatorname{ArF}(6.4 \mathrm{eV}$ per photon) mode. The half-width of the laser pulse is $20( \pm 2)$ ns with a 2 -ns rise/fall time and a maximum laser energy of $300 \mathrm{~mJ}$. The laser output cross section of $2.8 \times 1.2 \mathrm{~cm}^{2}$ is increased to $2.8 \times 2.8 \mathrm{~cm}^{2}$ using a lens system of fused silica cylindrical plano-convex and plano-concave lenses in order to increase the plasma filling fraction of the vacuum chamber. The laser beam enters a 5 -cm-diameter by

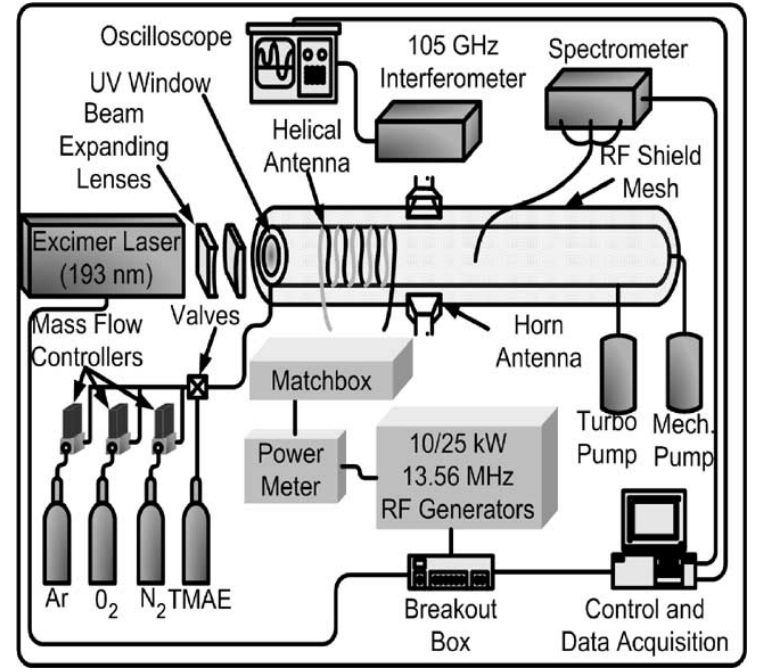

Fig. 1. Schematic of the experimental arrangement of the laser-initiated and RF sustained plasma. The lens system is used to modify the laser foot print cross section to $2.8 \times 2.8 \mathrm{~cm}^{2}$. The RF coil has not been energized in this experiment but will be utilized in future experiments.

150-cm-long Pyrex plasma chamber through a 2.8-cm-diameter Suprasil quartz window ( $98 \%$ transparency at $193 \mathrm{~nm}$ ) at one end. Laser energy passing through the UV window is measured using an energy meter SCIENTECH (Astral AD30). In order to account for the laser attenuation by the UV window, the UV window is placed in front of the energy meter. A laser fluence of $6 \mathrm{~mJ} / \mathrm{cm}^{2}$ is maintained. Gas mass flow controllers along with a swirl gas injection system are also located at this end as shown in Fig. 1. The plasma chamber is pumped down to a base pressure of $10^{-6}$ torr using a turbomolecular pump. In the evacuated chamber, the TMAE seed gas pressure is raised to optimum values of 4-50 mtorr and then the air or noble gas pressure is raised slowly over a minute to 760 torr.

TMAE $\left(\mathrm{C}_{10} \mathrm{H}_{24} \mathrm{~N}_{4}\right)$, a large molecule (molecular weight $=$ 200.3 ), is chosen for two reasons. First, the vertical ionization potential of TMAE is $6.1 \mathrm{eV}$ [16], much lower than that of the background gases. Secondly, the TMAE molecule has a large photoionization cross section for ultraviolet radiation and, therefore, a large volume plasma can be easily created. The continuous UV beamline photoionization quantum efficiency of TMAE at $193 \mathrm{~nm}$ is $30 \%$ [17]. In addition, TMAE is also a strong electron donor. Bright chemiluminescence associated with the reaction of TMAE and air in the gas phase is very long lived and is observable even after 30 min under some conditions [18]. We have also found that the room temperature vapor arising from the liquid form of TMAE is compatible with air over long times and that it is quite viable for efficient laser ionization in air for 10 min after introduction. Thus TMAE can be considered as a seed gas in air that can be efficiently ionized by a UV laser or a less expensive flashtube.

\section{B. Millimeter-Wave Interferometry}

A 105-GHz (QBY-1A10UW, Quinstar Technology) quadrature-phase, $\mathrm{mm}$-wave interferometer is used to characterize the temporal development of plasma during and following the application of the 20-ns laser pulse. The interferometer works in the Mach-Zehnder configuration, in which the plasma is in one 
arm of the two-beam interferometer. In low pressure discharges where the wave frequency $(\omega)$ is much greater than the plasma frequency $\left(\omega_{p}\right)$ and the collision frequency $(\nu)$, a linear relationship exists between the plasma density and the phase shift [19]. In this case, the wave suffers little or no attenuation as it travels through the plasma. However, at high gas pressures, where the collision frequency is comparable to both plasma and wave frequencies $\left(\nu \geq \omega \sim \omega_{p}\right)$, the wave propagating through the plasma arm undergoes phase change as well as strong attenuation. The wave attenuation is caused by the presence of high density as well as high collisionality. In this case the measured plasma density has a complex dependence on wave phase as well as amplitude changes. In order to calculate the refractive and dissipative properties, we consider an electromagnetic wave propagating in an infinite, uniform, collisional plasma. In this model, described in detail in [15], electron motion is induced by the electromagnetic wave and the ions are assumed to form a stationary background. Even though the laser ionization rate is very large, the assumptions for the model remain valid since time scale for change in plasma characteristics $(\tau \approx 10-100 \mathrm{~ns})$ will be much larger than the wave propagation time through the plasma and the fast response of the detector diode and the oscilloscope $(\tau \approx 2 \mathrm{~ns})$. Moreover, most of the density variation and recombination analyses were carried out during the plasma decay in the absence of an ionizing source. The complex dielectric constant yields a plane wave phase $\left(\beta_{p}\right)$ and attenuation $\left(\alpha_{p}\right)$ coefficients in the plasma as,

$$
\begin{aligned}
& \beta_{p}=\frac{\omega}{c}\left\{\frac{1}{2}\left(1-\frac{\omega_{p}^{2}}{\omega^{2}+\nu^{2}}\right)\right. \\
&+\left.\frac{1}{2}\left[\left(1-\frac{\omega_{p}^{2}}{\omega^{2}+\nu^{2}}\right)^{2}+\left(\frac{\omega_{p}^{2}}{\omega^{2}+\nu^{2}} \frac{\nu^{2}}{\omega}\right)^{2}\right]^{\frac{1}{2}}\right\}^{\frac{1}{2}} \\
& \alpha_{p}=\frac{\omega}{c}\left\{-\frac{1}{2}\left(1-\frac{\omega_{p}^{2}}{\omega^{2}+\nu^{2}}\right)\right. \\
&\left.+\frac{1}{2}\left[\left(1-\frac{\omega_{p}^{2}}{\omega^{2}+\nu^{2}}\right)^{2}+\left(\frac{\omega_{p}^{2}}{\omega^{2}+\nu^{2}} \frac{\nu}{\omega}\right)^{2}\right]^{\frac{1}{2}}\right\}^{\frac{1}{2}} .
\end{aligned}
$$

Assuming a plasma slab of uniform density profile, the total change in phase and amplitude for interferometric signal are given as

$$
\Delta \varphi=\int_{0}^{d}\left(\beta_{0}-\beta_{p}\right) d r ; \quad \Delta A=\int_{0}^{d}\left(\alpha_{0}-\alpha_{p}\right) d r
$$

where $\beta_{0}$ and $\alpha_{0}$ are the free space phase and attenuation coefficients. Simultaneous solutions of the plasma density $n_{e}$ and collision frequency $\nu$ are obtained from the experimentally measured $\Delta \varphi$ and $\Delta A$ values. This density measurement technique has also been verified by our group by comparison with Langmuir probe measurements in a high-density, collisional helicon plasma [20].

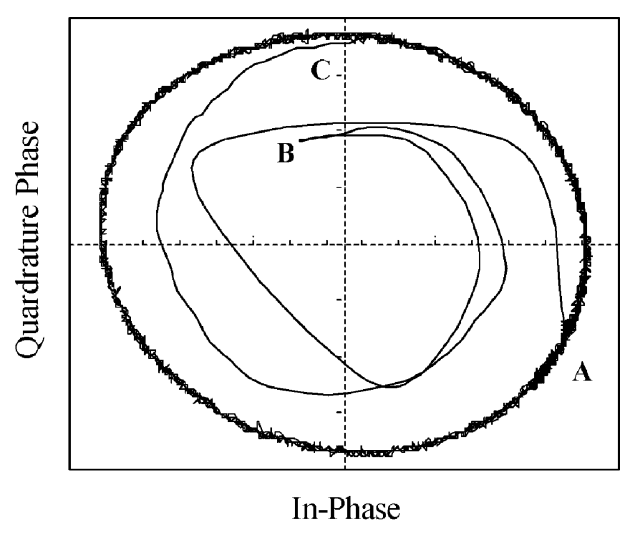

Fig. 2. Interferometer trace showing the phase and amplitude variation for a 35 mtorr TMAE plasma after the application of the laser pulse reaching a maximum line-average plasma density of $4 \times 10^{13} \mathrm{~cm}^{-3}(A \rightarrow B)$, followed by the plasma decay $(B \rightarrow C \rightarrow A)$ at a distance of $20 \mathrm{~cm}$ from the laser window. The outside circle represents the vacuum phase variation.

The interferometer works by using an I-Q (In-phase and Quadrature phase) mixer to obtain the phase and amplitude change of the $105-\mathrm{GHz} \mathrm{mm}$-wave signal that passes through the plasma. A fast digital storage oscilloscope with advanced triggering capabilities (LeCroy 9354 series, $2 \mathrm{GSa} / \mathrm{s}$ ) and GPIB inteRFace is used to transfer detector diode signals to the computer. The entire interferometer assembly is enclosed in a conducting box with slots for waveguides. We placed the $\mathrm{mm}$ wave antenna in both far and near field location and checked that the effect of $\mathrm{mm}$ wave diffraction due to plasma transverse $(d=2.8 \mathrm{~cm})$ dimension was insignificant. In order to minimize the noise level in the interferometer signal, semi-rigid co-axial cables with very efficient shielding $(\geq 90 \mathrm{~dB}$, Times Microwave Systems) have been used. An axial density scan is obtained by varying the position of the interferometer along the $z$ axis. The interferometer trace in Fig. 2 is shown as a function of time as the 35 mtorr TMAE plasma formed by the application of $20 \mathrm{~ns}$ laser pulse decays. Since the laser intensity is uniform $(\Delta \mathrm{I} / \mathrm{I} \leq 10 \%)$ over the $2.8 \mathrm{~cm}$ diameter and recombination processes are found to dominate diffusion processes [10], a uniform radial plasma density profile is assumed. A numerical program written in MATLAB solves simultaneously for both phase and attenuation data to obtain the average plasma density as well as the effective collision frequency.

The temporal plasma loss rate is obtained by taking the time derivative of density. Plasma lifetime after the application of the laser pulse is critical for an efficient transition to RF inductive wave coupling to sustain the plasma. The nature of the dominant loss mechanisms can be obtained from the temporal plot of plasma density where each point on the interferometer trace corresponds to the instantaneous line-average plasma density. By examining pure TMAE decay and then evaluating the decay in the presence of high-pressure individual air components and noble gases and as an ensemble, the key mechanisms affecting plasma lifetime can be isolated and determined.

\section{Spectroscopy}

Optical emission spectroscopy is also used to characterize the temporal evolution of TMAE plasma in the presence of background gases. Plasma emission passes through a high-quality 


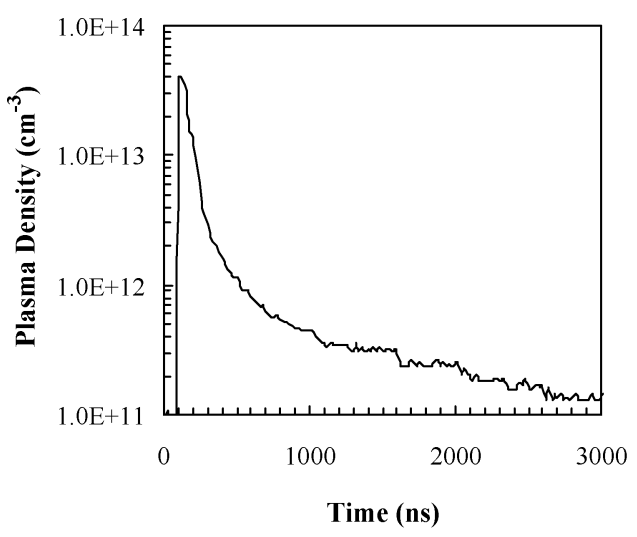

Fig. 3. Temporal plot of plasma density at 35 mtorr TMAE corresponding to interferometer trace shown Fig. 2.

UV (200-800 nm) fiber-optic bundle into a monochromator, and is then detected by a photomultiplier tube (PMT). A UV cutoff filter $(<300 \mathrm{~nm})$ is used in front of the fiber-optic bundle to eliminate the scattered $193 \mathrm{~nm}$ high-power source laser pulse that can saturate the PMT. It utilizes a $500 \mathrm{~mm}$ focal length monochromator (Acton Research SpectraPro-500i: Model SP-558) with a $1200 \mathrm{~g} / \mathrm{mm}$ grating and a high resolution of $0.05 \mathrm{~nm}$ at $435.8 \mathrm{~nm}$ and $10-\mu \mathrm{m}$ slit.

A control program written in LabView is used to obtain the time resolved emission wave spectrum. The program controls the triggering of the laser source, the optical system and the fast digital oscilloscope for data acquisition. The flexibility of operation, in controlling the various measurement parameters such as integration window referenced to laser pulse and averaging over 200 laser pulses, allows a precise measurement of wave spectrum. The life-time of super-excited TMAE states with and without the background gas can be measured accurately by the temporal evolution of TMAE plasma emission lines.

\section{RESULTS AND DISCUSSION}

\section{A. Plasma Density}

A large volume ( $~ 500 \mathrm{cc})$, high density $\left(n_{e} \geq 10^{13} / \mathrm{cm}^{3}\right)$ TMAE plasma is created by $193 \mathrm{~nm}$ laser in a plasma chamber. The plasma chamber is evacuated to a base pressure of $10^{-6}$ torr using a turbo-molecular pump. The TMAE pressure is raised to the desired value (4-50 mtorr) in this evacuated chamber by opening a TMAE liquid filled bottle at room temperature, while continuing to pump with the turbo-molecular pump and a dry backup pump at a slow rate. In order to maintain the TMAE vapor pressure above 30 mtorr in the chamber while continuing to pump at slow rate, hot air is blown on to the metal TMAE bottle. Experimentally determined temporal phase and amplitude change data are used simultaneously to solve for the temporal variation in plasma density and collision frequency.

The interferometer trace showing the phase and amplitude variation for a 35 mtorr seed TMAE plasma is presented in Fig. 2 and the temporal plot of the plasma density corresponding to the interferometer trace is shown in Fig. 3. The outside circle represents the phase variation for the vacuum conditions. The onset of plasma follows the path $A \rightarrow B$. The plasma formed by the application of the $20 \mathrm{~ns}$ laser pulse reaches the peak line-average plasma density value of $4 \times 10^{13} \mathrm{~cm}^{-3}$ at $z=20 \mathrm{~cm}$

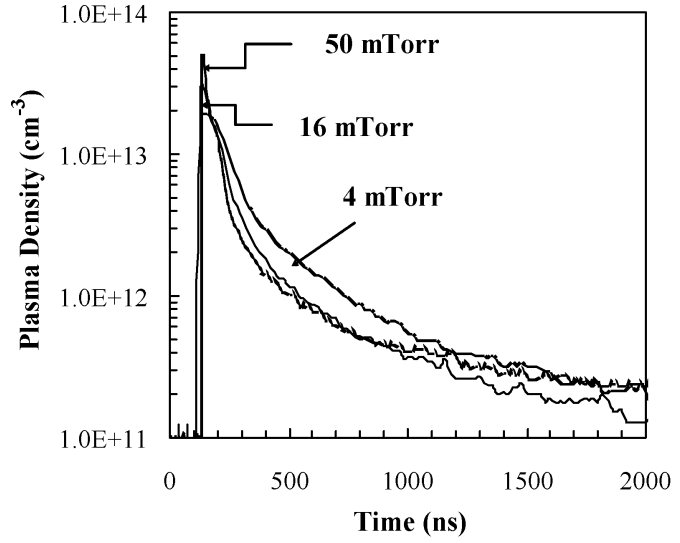

Fig. 4. TMAE plasma density versus time plot for at different TMAE vapor pressures.

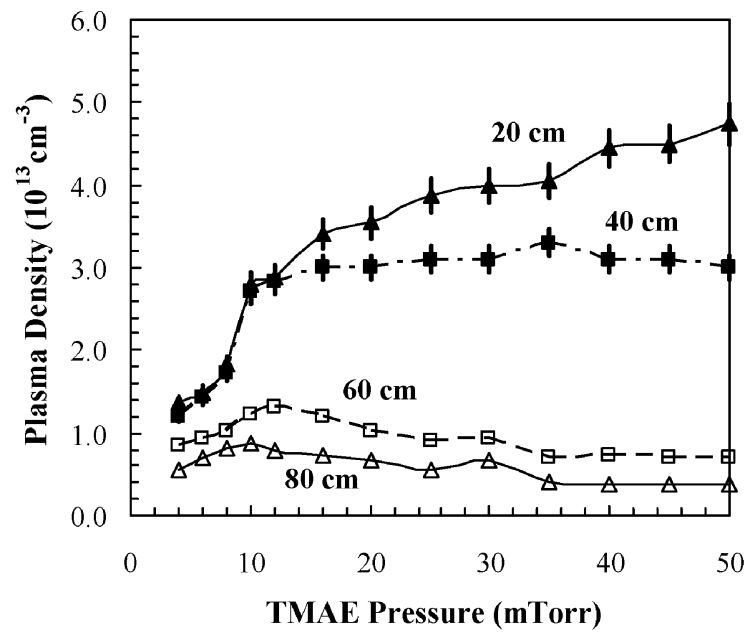

Fig. 5. TMAE plasma density versus TMAE vapor pressure at axial distances measured from the laser window for a fluence of $6 \mathrm{~mJ} / \mathrm{cm}^{2}$

from the Suprasil window. The TMAE plasma decays temporally along the path $B \rightarrow C \rightarrow A$. In Fig. 4, the temporal plot of the TMAE plasma density for 4,16 , and 50 mtorr TMAE vapor pressures is shown. The temporal density plot starts at $t=0$ and is referenced to the end of the 20-ns laser pulse. It should be noted that the peak plasma density occurs fairly late in time $(t=$ $140 \pm 10 \mathrm{~ns}$ ) after the application of laser pulse. Optical emission data also shows the presence of small (two-orders-of-magnitude lower) direct ionization process during the laser pulse. However, the initial low density plasma $\left(<10^{11} \mathrm{~cm}^{-3}\right)$ produced by direct ionization can not be accurately measured by the $105-\mathrm{GHz}$ interferometer. It is also observed that the plasma density increases with vapor pressure, while the plasma density decay is more rapid at higher vapor pressures. Note that during the early times for about $100 \mathrm{~ns}$ after the formation of plasma $(t=240 \mathrm{~ns})$, the density decay depends on the initial density and the two-body process involving electron-ion recombination plays the dominant role. Following the initial decay, the plasma density approaches comparable values for $t \geq 240 \mathrm{~ns}$. For times $>240 \mathrm{~ns}$, the density decay curves are similar for both 16 and 50 mtorr cases. In contrast, the density decay curve for 4 mtorr shows a slower decay rate for this time interval.

The plasma density for these different TMAE vapor pressures for $20-80 \mathrm{~cm}$ axial positions from the laser window is shown in 


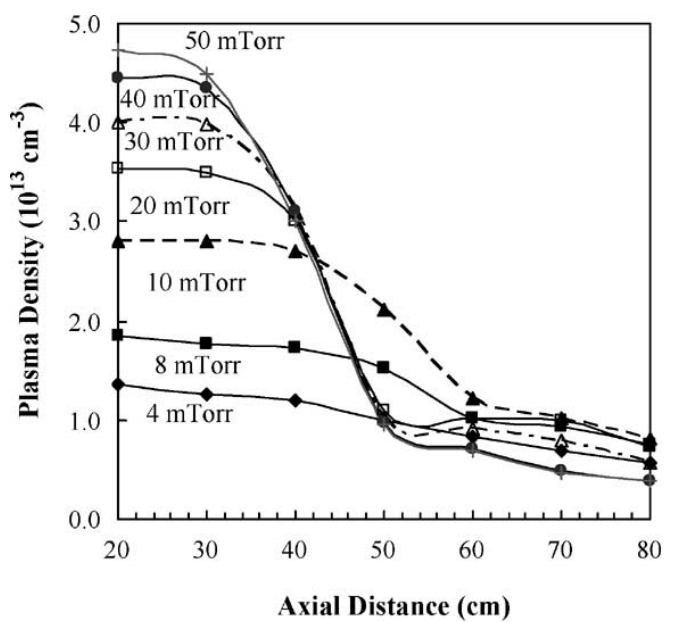

Fig. 6. Axial density plot for various TMAE vapor pressures for a laser fluence of $6 \mathrm{~mJ} / \mathrm{cm}^{2}$.

Fig. 5. The corresponding axial density decay plot is shown in Fig. 6. The laser flux for these measurements is held constant at $6 \mathrm{~mJ} / \mathrm{cm}^{2}$. The size of the vertical bar shown in Fig. 5 for axial distance $z=20 \mathrm{~cm}$ from the laser window represents the percent variation $(5 \%)$ over 10 shots. It is also shown in the plot that after an initial increase in the peak TMAE plasma density with increasing vapor pressure, the increase in peak plasma density with pressure is small above 40 mtorr. At $20 \mathrm{~cm}$ from the Suprasil window, there is a two-fold increase in the line-average peak density from $1.4 \times 10^{13} \mathrm{~cm}^{-3}$ at 4 mtorr to $2.8 \times 10^{13} \mathrm{~cm}^{-3}$ at 10 mtorr, and from $4.5 \times 10^{13} \mathrm{~cm}^{-3}$ at 40 mtorr it increases slightly to $4.7 \times 10^{13} \mathrm{~cm}^{-3}$ at 50 mtorr. The axial plasma density plot in Fig. 6 reveals a sharp axial plasma density decay for higher vapor pressure plasmas. The fractional peak plasma density at an $80 \mathrm{~cm}$ axial location with respect to its value at $20 \mathrm{~cm}$ is $40 \%, 30 \%, 14 \%$, and $8 \%$ for the $4,10,30$ and 50 mtorr cases, respectively. The sharp drop in axial plasma density for higher TMAE vapor pressure can be attributed to the enhanced laser absorption nearer the Suprasil window at higher pressures.

In order to study the effect of background gases on the TMAE plasma formation and decay characteristics in an evacuated chamber, the TMAE pressure is raised to an optimal pressure of 16 mtorr and then the background gas pressure is increased slowly to 760 torr. A temporal plot of the TMAE plasma density in the presence of different background gases is shown in Fig. 7. A laser fluence of $6 \mathrm{~mJ} / \mathrm{cm}^{2}$ is maintained. The temporal variation of 16 mtorr of pure TMAE plasma density is also shown in the plot for reference. The peak density of pure TMAE plasma is $3.2 \times 10^{13} \mathrm{~cm}^{-3}$ at $t=140 \mathrm{~ns}$. In the presence of 760 torr of noble gases such as helium and argon, the TMAE peak plasma density is reduced to $2.9 \times 10^{13} \mathrm{~cm}^{-3}$ and $2.3 \times 10^{13} \mathrm{~cm}^{-3}$, respectively. It corresponds to a density reduction of $10 \%$ for helium and $30 \%$ for an argon background gas. It is also observed that a high density $\left(>10^{12} \mathrm{~cm}^{-3}\right)$ plasma is maintained in the presence of noble background gases for over $2 \mu \mathrm{s}$. Since the background gas is at atmospheric pressure with neutral particle densities $\sim 2.5 \times 10^{19} \mathrm{~cm}^{-3}$, the effect of three-body recombination involving a neutral as the third particle becomes an important factor. In the experiment with room temperature air constituents as the background

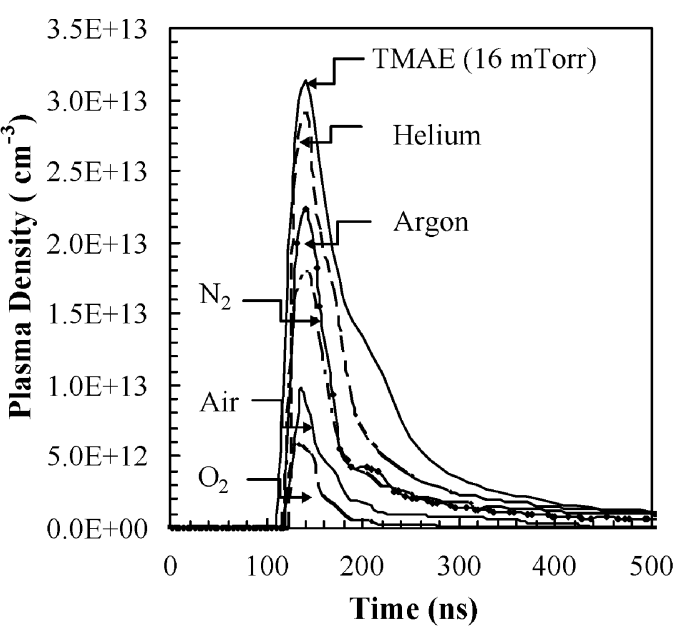

Fig. 7. 16-mtorr TMAE plasma density versus time plot in the presence of background gases at 760 torr.

gas, the effect of electron attachment is evident. The peak TMAE plasma densities obtained in the presence of 760 torr of nitrogen, oxygen and air are $1.8 \times 10^{13} \mathrm{~cm}^{-3}, 5.8 \times 10^{12} \mathrm{~cm}^{-3}$ and $9.8 \times 10^{12} \mathrm{~cm}^{-3}$, respectively. In addition, a TMAE plasma density $\geq 5 \times 10^{11} \mathrm{~cm}^{-3}$ can be maintained in atmospheric air for $t \leq 0.3 \mu \mathrm{s}$. This is long enough so that RF power can be coupled to the seed plasmas efficiently [7]. We also observe that the seed TMAE vapor remains viable for large-volume ( $\sim 500 \mathrm{cc})$ and high-density $\left(10^{13} \mathrm{~cm}^{-3}\right)$ laser ionization in air for $t \leq 10 \mathrm{~min}$.

\section{B. Plasma Decay}

The pure TMAE plasma decays mainly through two-body electron-ion recombination, three-body recombination with either an electron or a neutral as the third particle and via electron attachment to oxygen. Let us consider the temporal decay of average plasma density $\left\langle n_{e}(t, z)\right\rangle d$ at a given axial location written as $n_{e}$. In the absence of an ionizing source, the plasma decay can be described by the continuity equation as [6], [9], [13], [14],

$$
\frac{d n_{e}}{d t}=-D_{a} \nabla^{2} n_{e}-\alpha_{r} n_{e}^{2}-\beta_{r} n_{r} n_{e}^{2}-\kappa_{a} n_{e} n_{g}^{2}
$$

Here, $D_{a}$ is the ambipolar diffusion term [21], $\alpha_{r}\left(\mathrm{~cm}^{3} / \mathrm{s}\right)$ is the two-body (electron-ion) recombination coefficient. Here $\beta_{r}\left(\mathrm{~cm}^{6} / \mathrm{s}\right)$ is the three-body (electron-ion) recombination coefficient and $n_{r}$ is the particle density of the third species. This three-body recombination process can involve either a neutral atom $\left(\beta_{g} ; n_{g}\right)$ or an electron $\left(\beta_{e} ; n_{e}\right) . n_{g}$ is the neutral particle density of the background gas and $\kappa_{a}\left(\mathrm{~cm}^{6} / \mathrm{s}\right)$ is the three-body electron attachment rate coefficient for the process $e+\mathrm{O}_{2}+$ $\mathrm{M} \rightarrow \mathrm{O}_{2}^{-}+\mathrm{M}\left(\mathrm{M}=\mathrm{O}_{2}, \mathrm{~N}_{2}\right)$. The diffusive loss in a pure TMAE plasma after the application of the $20 \mathrm{~ns}$ laser pulse is small on a microsecond time scale in agreement with prior measurements by our group [10].

Two-body recombination plays a larger role in density decay for low pressure discharges whereas the contribution of three-body recombination process becomes important only either at higher density or at higher gas pressures. The rate coefficient for the three-body recombination process where the third 


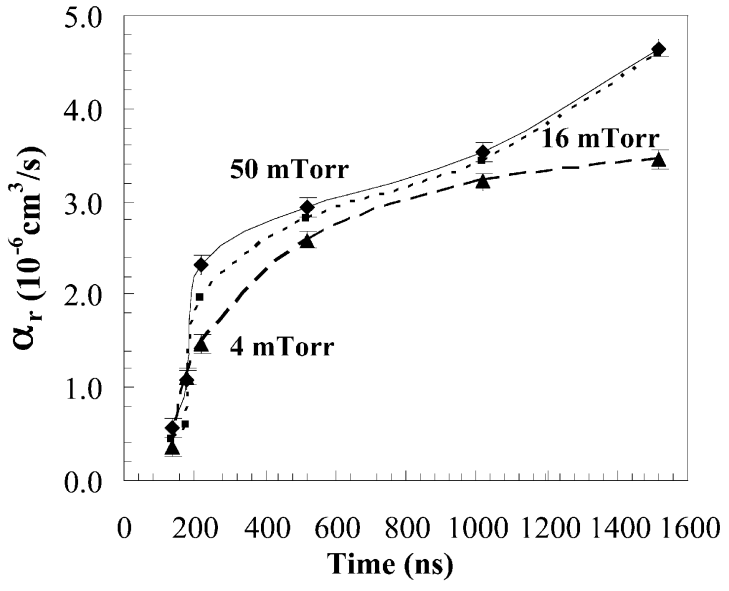

Fig. 8. Temporal plot of recombination coefficients for TMAE plasma.

body is an electron, i.e., $A^{*}+e+e \rightarrow A^{*}$ where $A^{*}$ is an excited neutral, is given by $\beta_{e} \approx 1.64 \times 10^{-9}\{\mathrm{~T}(\mathrm{~K})\}^{-9 / 2} \mathrm{~cm}^{6} / \mathrm{s}$ [22]. For electron densities of $n_{e} \sim 10^{13} \mathrm{~cm}^{-3}$ at room temperature, the loss factor $\beta_{e} n_{e}$ is $1.2 \times 10^{-7} \mathrm{~cm}^{3} / \mathrm{s}$. The neutral-stabilized, electron-ion collisional recombination rate for the process, $A^{+}+e+B \rightarrow A^{*}+B$, where $B$ is a neutral atom is given as [23], [24]

$$
\beta_{g} \approx 6 \times 10^{-27}\left(\frac{300}{\mathrm{~T}(\mathrm{~K})}\right)^{1.5}\left(\mathrm{~cm}^{6} / \mathrm{s}\right) .
$$

For a pure TMAE plasma at a maximum pressure of 50 mtorr at $300 \mathrm{~K},\left(n_{g} \approx 1.6 \times 10^{15} \mathrm{~cm}^{-3}\right)$, the loss factor, $\beta_{g} n_{g}=$ $9.6 \times 10^{-12} \mathrm{~cm}^{3} / \mathrm{s}$, can be neglected. However, at 760 torr where the neutral particle density, $n_{g}=2.45 \times 10^{19} \mathrm{~cm}^{-3}$, the loss factor $\beta_{g} n_{g}=1.5 \times 10^{-7} \mathrm{~cm}^{3} / \mathrm{s}$ becomes important.

1) TMAE Plasma: Since the TMAE molecule is a strong electron donor [16], [17], the electron attachment process in a pure TMAE plasma is very small and is neglected. As mentioned earlier for TMAE partial pressures of 4-50 mtorr, the contributions of three-body recombination loss processes $A^{+}+$ $e+e \rightarrow A^{*}+e$ and $A^{+}+e+B \rightarrow A^{*}+B$ are an order of magnitude smaller and are neglected in this analysis. Therefore, for a temporally decaying TMAE plasma, the continuity equation (4) takes the form

$$
\frac{d n_{e}}{d t}=-\alpha_{r} n_{e}^{2}
$$

The recombination coefficient for a TMAE plasma can be measured from the temporal plot of plasma density. Numerical solution of (6) is obtained by determining the electron densities $n_{e 1}$ and $n_{e 2}$ at two closely-spaced measurement times $t_{1}$ and $t_{2}$, respectively. It is given as

$$
\alpha_{r}=\frac{\left(\frac{1}{n_{e 2}}-\frac{1}{n_{e 1}}\right)}{\left(t_{2}-t_{1}\right)} .
$$

Using the data from the temporal density plot in Fig. 4, a temporal plot of $\alpha_{r}$ after the application of laser pulse is shown in Fig. 8. As shown in the plot, the recombination coefficient increases with time. For a 10 mtorr TMAE plasma, $\alpha_{r}$ increases from $4.4 \times 10^{-7} \mathrm{~cm}^{3} / \mathrm{s}$ at $140 \mathrm{~ns}$ to $4.5 \times 10^{-6} \mathrm{~cm}^{3} / \mathrm{s}$ at 1500 ns. It also shows a more rapid initial increase in $\alpha_{r}$ for higher TMAE vapor pressures. These TMAE plasma density decay results obtained from interferometric measurement are in agreement with the previous late in time Langmuir probe measurements by our group [10].

This increase in $\alpha_{r}$ with time can be explained in terms of a delayed ionization process [10], [25], [26]. Single photon ionization of large molecules does not necessarily result in prompt ionization, even though the photon energy is above the vertical ionization potential of the molecule [25]. The incident photons absorbed by TMAE molecules produce super-excited TMAE** neutrals. The super-excited TMAE ${ }^{* *}$ molecules store energy in the vibrational states and it is the slow, diffusive-like transfer of this energy to the departing electrons that determines the ionization rate [26] as has been observed for larger molecules such as $C_{60}$ and metal clusters [30]-[34]. This process is known as delayed ionization and plays an important role in the TMAE plasma formation and subsequent decay process. These superexcited neutrals decay by either electron emission (delayed ionization) or dissociate through the processes

$$
A B+h \nu \rightarrow A B^{* *} \rightarrow\left\{\begin{array}{l}
A B^{+}+e \text { (delayed ionization) } \\
A+B \quad \text { (dissociation) }
\end{array}\right.
$$

The process of delayed ionization can be incorporated in the temporal TMAE plasma density decay as [10],

$$
\frac{d n_{e}}{d t}=-\alpha^{\prime} n_{e}^{2}+D(t)
$$

where $D(t)$ is the delayed ionization coefficient. Substituting $d n_{e} / d t=-\alpha_{r} n_{e}^{2}$ from (6), we obtain $D(t)=\left(\alpha^{\prime}-\alpha_{r}\right) n_{e}^{2}$. It implies that $\alpha^{\prime}-\alpha_{r}$ is the change in recombination due to the delayed ionization process.

\section{2) Background Gas:}

a) Noble gases: In the presence of noble gases at 760 torr, three-body recombination involving neutrals as the third particle becomes significant. Neglecting electron attachment, (4) can be expressed as,

$$
\frac{\partial n_{e}}{\partial t}=-\alpha_{r} n_{e}^{2}-\beta_{g} n_{g} n_{e}^{2}=-\left(\alpha_{r}+\beta_{g} n_{g}\right) n_{e}^{2} .
$$

Here $\alpha_{r}$ represents the recombination losses for pure TMAE plasma described in (4) and $\beta_{g}$ is the loss due to three-body recombination where the third body is a neutral atom. In order to determine $\beta_{g}$ for TMAE in the presence of helium and argon, a numerical derivative of the TMAE plasma density temporal plot shown in Fig. 7 is obtained. Using the recombination coefficient, $\alpha_{r}$, already obtained for pure TMAE (Fig. 8) along with the neutral particle gas density, $n_{g}$, (9) is numerically solved in time to determine $\beta_{g}$. A plot of the three-body recombination rate $\beta_{g}$, is presented in Fig. 9. Since the three-body recombination process depends only on the neutral gas density, maintained at 760 torr during the experiment, we observe a very small temporal variation in $\beta_{g}$. The small variation $(\approx 5 \%)$ is within statistical error. In this experiment, the three-body recombination rate coefficients for TMAE in the presence of helium and argon are determined to be $\beta_{g}(\mathrm{He})=(4.35 \pm 0.7) \times 10^{-26} \mathrm{~cm}^{6} / \mathrm{s}$ and $\beta_{g}(\mathrm{Ar})=(9.5 \pm 0.8) \times 10^{-26} \mathrm{~cm}^{6} / \mathrm{s}$, respectively. The values 


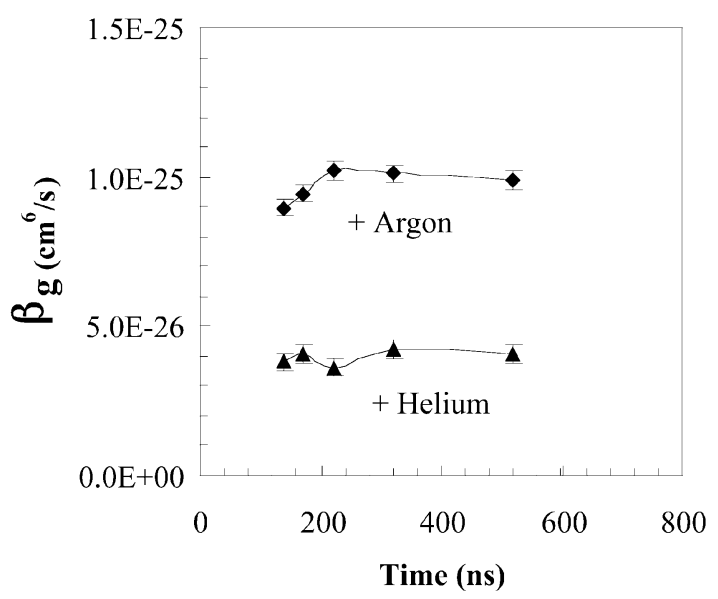

Fig. 9. Three-body recombination rate coefficients for TMAE plasma in the presence of helium and argon at 760 torr.

we obtain are comparable to published collisional three-body recombination rates for singly ionized plasma [27].

b) Air constituent gases: In atmospheric pressure air at room temperature, the dominant density loss mechanism in a TMAE plasma will be electron attachment to oxygen through the three-body process, $e+\mathrm{O}_{2}+\mathrm{M} \rightarrow \mathrm{O}_{2}^{-}+\mathrm{M}\left(\mathrm{M}=\mathrm{O}_{2}, \mathrm{~N}_{2}\right)$. Negative oxygen ions are removed by ionic recombination and this results in a significant reduction in the plasma density and life-time. The density decay (4) for this case is written as

$$
\frac{d n_{e}}{d t}=-\alpha_{r} n_{e}^{2}-\beta_{g} n_{g} n_{e}^{2}-\kappa_{a} n_{e} n_{g}^{2}
$$

Here $\beta_{g}$ is the three-body recombination rate coefficient with either oxygen or nitrogen as the third species and $\kappa_{a}$ is the electron attachment rate coefficient for oxygen and nitrogen. Based on the classical diffusion model that includes the elastic scattering of electrons by diatomic molecules, the three-body rate at room temperature is taken as $\beta_{g}=10^{-26} \mathrm{~cm}^{6} / \mathrm{s}$ [24], [28]. The differences in $\beta_{g}$ values for homonuclear diatomic molecules such as oxygen, nitrogen and hydrogen are small due to the absence of permanent dipole moments [24].

A numerical solution of (10) is obtained for the electron attachment coefficient, $\kappa_{a}$, by using numerical differentiation of the temporal decay of the TMAE plasma density in the presence of air constituents (Fig. 7) along with the known effective two-body recombination coefficient, $\alpha_{r}$, for pure TMAE (Fig. 8). A temporal plot of electron attachment rate coefficient, $\kappa_{a}$, for nitrogen, oxygen and air when they are individually added to TMAE is shown in Fig. 10. The electron attachment rate decreases temporally with the TMAE plasma density. This illustrates that the probability of electron attachment decreases with a decrease in the plasma density. In the presence of nitrogen at $t=140 n s$, the peak value of $\kappa_{a}\left(\mathrm{~N}_{2}\right)$ is $5.6 \times 10^{-32} \mathrm{~cm}^{6} / \mathrm{s}$. As a result, the nitrogen's subsequent contribution to the TMAE plasma loss in air is small. This is expected since nitrogen does not readily form a negative ion. The dominant plasma loss can be attributed to the presence of the oxygen [23]. However, for oxygen, the peak electron attachment rate coefficient $\kappa_{a}\left(\mathrm{O}_{2}\right)$ at $t=140 \mathrm{~ns}$, when the

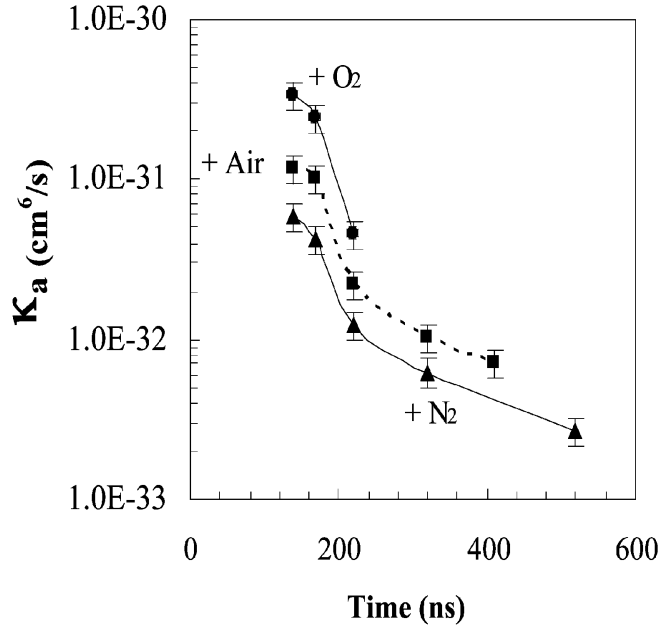

Fig. 10. Electron attachment rate coefficient for TMAE plasma in the presence of nitrogen, oxygen and air at 760 torr.

TMAE density is maximum, is $3.2 \times 10^{-31} \mathrm{~cm}^{6} / \mathrm{s}$. It is almost an order-of-magnitude higher than nitrogen. In the presence of atmospheric air, the electron attachment rate to oxygen is $1.1 \times 10^{-31} \mathrm{~cm}^{6} / \mathrm{s}$. These electron attachment rate coefficients, for TMAE plasmas in nitrogen, oxygen and air are lower by almost an order-of-magnitude than the values obtained for the process $e+\mathrm{O}_{2}+\mathrm{M} \rightarrow \mathrm{O}_{2}^{-}+\mathrm{M}\left(\mathrm{M}=\mathrm{O}_{2}, \mathrm{~N}_{2}, \mathrm{H}_{2} \mathrm{O}\right)$ in room temperature air plasmas without TMAE [22]. This indicates that the process of delayed ionization of TMAE that has a much longer lifetime than the direct ionization gradually populates the emissive state and plays an important role in increasing the lifetime of the TMAE plasma for RF sustainment at lower power.

\section{Plasma Emission Spectroscopy}

The optical emission spectra of 193-nm laser-produced TMAE plasma is obtained using a high resolution monochromator. The entrance and exit slit widths were set at $2000 \mu \mathrm{m}$ to obtain a statistically large number of photon counts per acquisition. A wavelength scan of the emission spectrum from $300-650 \mathrm{~nm}$, with a step size of $4 \mathrm{~nm}$ and averaged over 200 laser pulses is obtained. A user-defined program written in Lab View provides the flexibility of arbitrary integration window size, accurate referencing of integration window with respect to the laser pulse, and improved statistics by averaging over a large number of laser pulses. The emission spectrum of 16 mtorr of pure TMAE plasma measured for two time windows: 1) $100 \mathrm{~ns}<t<600 \mathrm{~ns}$ and 2) $100 \mathrm{~ns}<t<1100 \mathrm{~ns}$, referenced to the laser pulse turn-on with the laser flux held constant at $6 \mathrm{~mJ} / \mathrm{cm}^{2}$ is shown in Fig. 11. The spectrum has maxima at 448 and $480 \mathrm{~nm}$. The $480 \mathrm{~nm}$ has been reported as a peak emission and corresponds to the first Rydberg state TMAE* (R1) with a 20 ns lifetime [29], [30].

In order to obtain the temporal evolution of the $480 \mathrm{~nm}$ line corresponding to the TMAE* (R1) state over $t \leq 800 \mathrm{ns,} \mathrm{slit}$ width of 500 microns along with a narrow integration window of $10 \mathrm{~ns}$ is used. Fig. 12 clearly shows that the peak of $480-\mathrm{nm}$ emission occurs fairly late in time $(\tau=140 \pm 10 \mathrm{~ns})$ after the application of 20-ns laser pulse. We also observe small (two- 


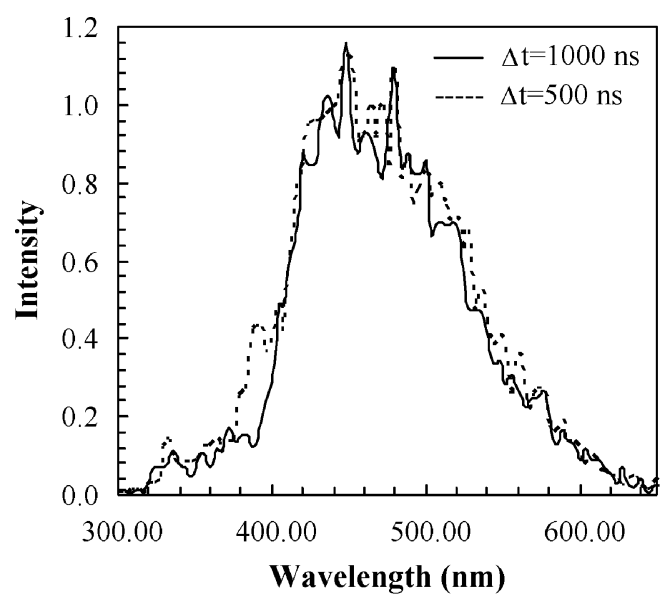

Fig. 11. Emission spectrum of 16 mtorr TMAE plasma measured for two time windows (a) $100 \mathrm{~ns}<t<600 \mathrm{~s}$ and (b) $100 \mathrm{~ns}<t<1100 \mathrm{~ns}$.

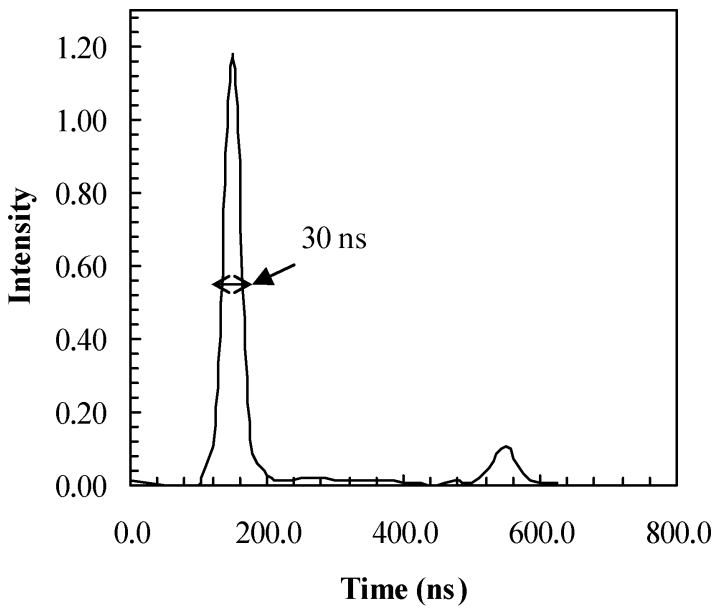

Fig. 12. Temporal evolution of 480-nm line corresponding to TMAE Rydberg states (R1) showing delayed ionization with super-excited state life-time of 140 $\pm 10 \mathrm{~ns}$.

orders-of-magnitude lower) 480-nm emission due to the direct ionization process during the laser pulse. In order to reference the plasma temporal emission to the turn-on of the laser pulse, the laser temporal profile was accurately measured by a fast UV photodiode (Hamamatsu S1226-18BQ with less than 10-ns rise time) using a 2-GHz Lecroy sampling oscilloscope. This late emission of the 480-nm peak can be interpreted in terms of the phenomenon of delayed ionization of TMAE.

The absence of direct ionization in TMAE is contrary to the traditional interpretation of the ionization process associated with small molecules. The process of ionization of small molecules is very direct and once the ionization energy is exceeded, free electrons depart on a femtosecond time scale [31]. However, for larger molecules such as $C_{60}$ and metal oxide clusters, the ionization is no longer prompt and there is a measurable time delay in the appearance of the electrons [31]-[34]. Schlag and Levin [25], [26] proposed that even though the photons provide the energy necessary to initiate electron removal, the actual departure of electrons and, hence, ionization is delayed.

Most of the photons absorbed by the TMAE molecules do not contribute to the direct ionization process. Even though our

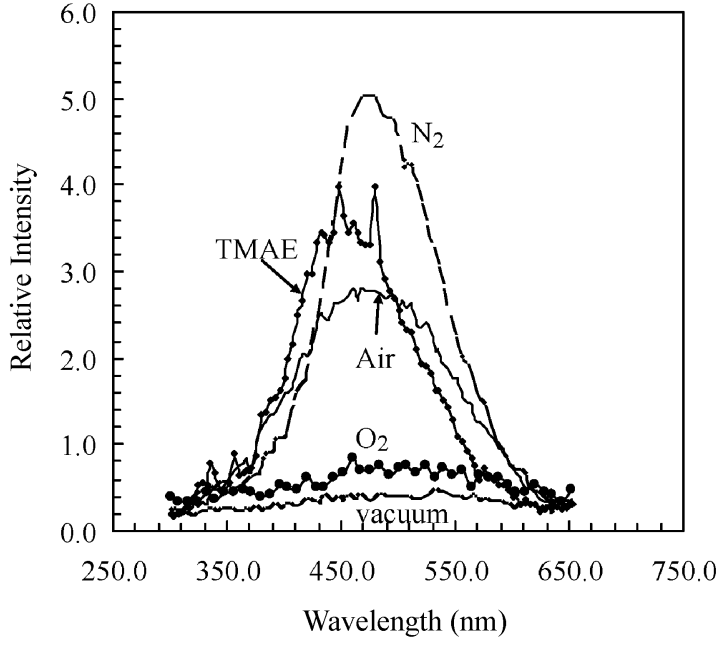

Fig. 13. Effect of atmospheric pressure background gases nitrogen, oxygen and air on the emission spectra of 16 mtorr TMAE plasma measured during the time window $100 \mathrm{~ns}<t<1100 \mathrm{~ns}$.

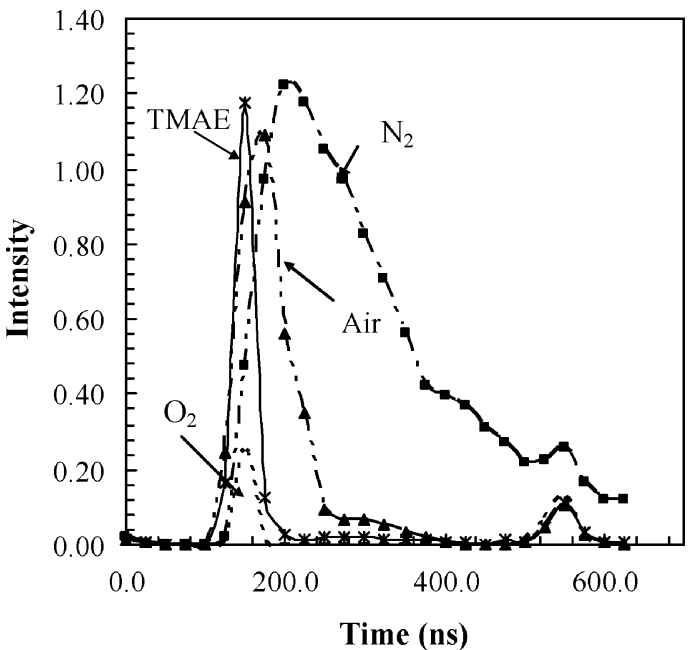

Fig. 14. Temporal evolution of $480 \mathrm{~nm}$ line corresponding to TMAE Rydberg states (R1) for 16 mtorr TMAE plasma in the presence of air constituent gases nitrogen, oxygen and air at 760 torr.

laser photon energy of $6.4 \mathrm{eV}$ is above the TMAE vertical ionization potential $(6.1 \mathrm{eV})$ [16], the experiment indicates that the additional energy of $0.3 \mathrm{eV}$ above the ionization potential is not sufficient to produce substantial direct ionization of the large TMAE molecule (molecular weight $=200.3$ ). Instead, these photons excite the neutrals to a super-excited state. These super-excited TMAE neutrals (TMAE**) store energy in the many degrees of freedom of the molecule and then transfer energy on a slower time scale to the departing free electrons. The delay in the peak 480-nm emission after the application of the laser pulse corresponds to the relaxation time of the super-excited state. From the temporal plot of the 480-nm emission, we obtain the relaxation time (the lifetime) of the super-excited state to be $\tau \cong 140 \pm 10$ ns. The lifetime of the first Rydberg state of TMAE given by the observed emission spectrum full-width at half-maximum (FWHM) is $30 \mathrm{~ns}$.

The wavelength scan of the emission spectra of 16 mtorr of TMAE in the presence of background gases is shown in Fig. 13. The corresponding temporal evolution of the 480-nm 
line is shown in Fig. 14. The emission spectrum increases in the presence of nitrogen as compared to the pure TMAE spectrum, whereas the peak emission drops significantly in the presence of oxygen and it is only slightly higher than the noise level. The decrease in plasma emission in the presence of oxygen can be explained in terms of the rapid quenching of TMAE plasma through the process of electron attachment to oxygen as is well known for air plasmas [18]. In addition, energy transfer between excited molecules and radicals followed by electron attachment to oxygen can also play an important role. This result is in agreement with the interferometric measurements of lower density and a shorter lifetime of TMAE plasma in the presence of room temperature oxygen. We observe a decrease in the plasma emission with atmospheric pressure air compared to TMAE alone. However, the plasma emission as well as plasma density measurement $\left(n_{e} \approx 10^{13} \mathrm{~cm}^{-3}\right)$ indicates that a high density $\left(>5 \times 10^{11} \mathrm{~cm}^{-3}\right)$ TMAE plasma in air can be maintained for $t \leq 0.3 \mu$ s such that efficient coupling of RF power can occur [9].

The increase in plasma emission, as shown in Fig. 13, due to the presence of nitrogen is on the higher wavelength side close to the 480-nm Rydberg line. In addition, we observe in Fig. 14 that the peak of the 480-nm line occurs $200 \mathrm{~ns}$ after the laser pulse and that the FWHM of the Rydberg emission process increases to $170 \mathrm{~ns}$. Since nitrogen does not react with TMAE and also does not absorb 193-nm photons, the enhancement of the emission intensity implies that the nitrogen molecules enhance the excitation of the TMAE** state, where energy is stored, during the application of laser pulse [10]. These highly excited TMAE $^{* *}$ states gradually decay by electron emission and populate the first Rydberg state through the process TMAE ${ }^{* *}+\mathrm{N}_{2} \rightarrow$ $\mathrm{TMAE}^{*}(\mathrm{R} 1)+\mathrm{N}_{2}$. This gradual population of the TMAE* $(\mathrm{R} 1)$ state and subsequent emission results in a fairly broad temporal profile of $480 \mathrm{~nm}$ emission.

\section{SUMMARY}

Experiments were performed to explore the feasibility of using UV laser preionization of an organic vapor TMAE seeded in atmospheric pressure air to initiate a discharge that can later be sustained by radiofrequency power by inductive wave coupling. A large volume ( $\sim 500 \mathrm{cc})$ and high-density $\left(\sim 10^{13} \mathrm{~cm}^{-3}\right.$ ) electrodeless plasma was created by laser photoionization of TMAE seeded in atmospheric air. The temporal evolution of TMAE plasma after the application of the 20-ns laser pulse was studied using a $105-\mathrm{GHz}$ mm-wave interferometer and plasma emission spectroscopy. The TMAE plasma loss parameters including two- and three-body recombination coefficients and the oxygen-electron attachment rate coefficient were determined for the first time for the future radiofrequency pulsed plasma sustainment experiments on a large test chamber. In addition high density $\left(n_{e} \geq 10^{12} \mathrm{~cm}^{-3}\right)$ TMAE plasma is maintained for more than $2 \mu$ s after the application of laser pulse in the presence of noble gases and nitrogen. The experiment showed that it is possible to create a large-volume ( $\sim 500 \mathrm{cc})$, high-density $\left(\sim 10^{13} \mathrm{~cm}^{-3}\right)$ TMAE plasma in 760 torr air and $n_{e} \geq 5 \times 10^{11} \mathrm{~cm}^{-3}$ can be maintained with $t \leq 0.3 \mu \mathrm{s}$. The electron attachment to oxygen at room temperature is found to be the dominant density loss mechanism in air for laser produced TMAE plasma. TMAE also remains chemically active and viable for high density photoionization in air for more than $10 \mathrm{~min}$. It is also shown in the experiment that the main process of TMAE photoionization is delayed ionization that occurs $140 \pm 10 \mathrm{~ns}$ after the laser pulse.

The results indicate that the radio frequency power budget required to initiate a high density $\left(10^{13} \mathrm{~cm}^{-3}\right)$ and large volume (500 cc) atmospheric pressure air discharge can be substantially reduced. Since the electron attachment to oxygen is found to be the dominant loss mechanism for TMAE plasma in air, we foresee an optimum electrodeless scenario where pulse injected TMAE in rapidly puffed heated air at $2000 \mathrm{~K}$ to reduce electron attachment to $\mathrm{O}_{2}$ is ionized by UV optical means that facilitates the efficient coupling of high power pulsed radiofrequency power to the plasma and reduces power requirements for high-density $\left(10^{13} \mathrm{~cm}^{-3}\right)$ air plasma for variety of applications.

\section{REFERENCES}

[1] P. Tsai, L. Wadsoworth, and J. R. Roth, "SuRFace modification of fabrics using a one-atmosphere glow discharge plasma to improve fabric wettability," Text. Res. J., vol. 67, p. 359, 1997.

[2] K. Kelly-Wintenberg, T. C. Montie, C. Brickman, J. R. Roth, A. K. Carr, K. Sorge, L. C. Wadsworth, and P. P. Y. Tsai, "Room temperature sterilization of suRFaces and fabrics with a one atmosphere uniform glow discharge plasma," J. Ind. Microbiol. Biotechnol., vol. 20, p. 69, 1998.

[3] M. Laroussi, "Interaction of microwaves with atmospheric pressure plasmas," Int. J. Infrared Millimeter Waves, vol. 16, p. 2069, 1995.

[4] - "Scattering of EM waves by a layer of air plasma surrounding a conducting cylinder," Int. J. Infrared Millimeter Waves, vol. 17, p. 2215, 1996.

[5] R. J. Vidmar, "On the use of atmospheric pressure plasmas as electromagnetic reflector and absorbers," IEEE Trans. Plasma Sci., vol. 18, p. 733, Aug. 1990.

[6] R. J. Vidmar and K. R.Kenneth R. Stalder, "Air chemistry and power to generate and sustain plasmas: Plasma lifetime calculations," in Proc. AIAA, 2003, pp. 1-8.

[7] S. Ramakrishnan and M. W. Rogozinski, "Properties of electric arc plasma for metal cutting," J. Appl. Phys. D, vol. 60, p. 2771, 1986.

[8] A. Schutze, J. Y. Young, S. E. Babayan, J. Park, G. S. Selwyn, and R. F. Hicks, "The atmospheric-pressure plasma jet: A review and comparison to other plasma sources," IEEE Trans. Plasma Sci., vol. 26, p. 1685, Dec. 1998.

[9] K. L. Kelly, J. E. Scharer, E. S. Paller, and G. Ding, "Laser ionization and radio frequency sustainment of high-pressure seeded plasmas," $J$. Appl. Phys., vol. 92, p. 698, 2002.

[10] G. Ding, J. E. Scharer, and K. Kelly, "Diagnostics and analyses of decay process in laser produced tetrakis(dimethyl-amino)ethylene plasma," Phys. Plasmas, vol. 8, p. 334, 2001.

[11] — "Effects of rapidly decaying plasmas on Langmuir probe measurements," J. Appl. Phys., vol. 84, p. 1236, 1998.

[12] K. Kelly, J. E. Scharer, G. Ding, M. Bettenhausen, and S. P. Kuo, "Microwave reflections from a vacuum ultraviolet laser produced plasma sheet," J. Appl. Phys., vol. 85, p. 63, 1999.

[13] K. R. Stalder, R. J. Vidmar, and D. J. Eckstrom, "Observations of strong microwave absorption in collisional plasmas with gradual density gradients," J. Appl. Phys., vol. 72, p. 5098, 1992.

[14] K. R. Stalder and D. J. Eckstrom, "Afterglow decay kinetics of nonuniform plasmas with cylindrical symmetry: Application to the measurement of electron decay in large photoionized plasmas in atmosphericpressure," J. Appl. Phys., vol. 72, p. 3917, 1992.

[15] K. Akhtar, J. Scharer, S. Tysk, and E. Kho, "Plasma interferometry at high pressures," Rev. Sci. Instrum., vol. 74, p. 996, 2003.

[16] Y. Nakato, M. Ozaki, A. Egawa, and H. Tsubomura, "Organic amino compounds with very low ionization potentials," Chem. Phys. Lett., vol. 9, no. 6, p. 615, 1971.

[17] R. A. Holroyd, J. M. Preses, C. L. Woody, and R. A. Johnson, "Measurement of the absorption length ans absolute quantum efficiency of TMAE and TEA from threshold to $120 \mathrm{~nm}$," Nucl. Instr. and Meth. Phys. Res., vol. A261, p. 440, 1987.

[18] S. Toby, P. A. Astheimer, and F. S. Toby, "Chemiluminescence in the gas phase reaction between tetrakis (dimethylamino) ethylene and oxygen," J. Photochem. Photobiol., vol. A67, p. 1, 1992. 
[19] I. H. Hutchinson, Principles of Plasma Diagnostics. Cambridge, U.K.: Cambridge Univ. Press, 2002, p. 114.

[20] S. M. Tysk, C. M. Denning, J. E. Scharer, and K. Akhtar, "Optical and wave measurements and modeling of helicon plasmas for a wide range of magnetic fields," Phys. Plasmas, vol. 11, no. 3, p. 878, Mar. 2004.

[21] M. A. Lieberman and A. J. Lichtenberg, Priniciples of Plasma Discharges and Materials Processing. New York: Wiley, 1994, p. 33.

[22] Y. P. Raizer, Gas Discharge Physics. Berlin, Germany: SpringerVerlag, 1991, p. 62.

[23] M. Capitelli, C. M. Ferreira, B. F. Gordiets, and A. I. Osipove, Plasma Kinetics in Atmospheric Gases. Berlin, Germany: Springer Verlag, 2000, p. 140.

[24] D. R. Bates, "Classical theory of electron-ion recombination in an ambient gas," J. Phys. B., vol. 13, p. 2587, 1980.

[25] E. W. Schlag and R. D. Levin, "Ionization, charge separation, charge recombination and electron transfer in large systems," J. Phys. Chem., vol. 96, p. 10608, 1992.

[26] R. D. Levin, "Separation of time scales in the dynamics of high molecular Rydberg states," Adv. Chem. Phys., vol. 101, p. 625, 1997.

[27] Y. B. Zel'dovich and Y. P. Raizer, Physics of Shock Waves and HighTemperature Hydrodynamic Phenomena. New York: Academic, 1966, vol. 1, p. 407.

[28] L. M. Biberman, V. S. Vorob'ev, and I. T. Yakubov, Kinetcis of Nonequilibrium Low-Temperature Plasmas. New York: Consultant Bureau, 1987, p. 412.

[29] M. Hori, K. Kimura, and H. Tsubomura, "The electronic spectrum and chemiluminescence of tetrakis-(dimethylamino)-ethylene(TDAE)," Spectrochimica Acta, vol. 24A, p. 1397, 1968.

[30] Y. Nakato, M. Ozaki, and H. Tsubomura, "Photoionization and rydberg states of tetraaminoethylenes," J. Phys. Chem., vol. 76, p. 2105, 1972.

[31] R. L. Platzman, Radiation Research, G. Silini, Ed. Amsterdam, The Netherlands: North-Holland, 1967.

[32] G. E. E. B. Campbell, G. Ulmer, and I. V. Hertel, "Delayed ionization of $C_{60}$ and $C_{70}$," Phys. Rev. Lett., vol. 67, p. 1986, 1991.

[33] P. Wurz, K. R. Lykke, M. J. Pellin, and D. M. Gruen, "Velocity distributions and photodissociation of neutral $C_{60}$ and $C_{70}$ clusters," J. Appl. Phys., vol. 70, p. 6647, 1991.

[34] F. Remacle and R. D. Levin, "Prompt and delayed ionization of large molecules," Phys. Lett. A, vol. 173, p. 284, 1993

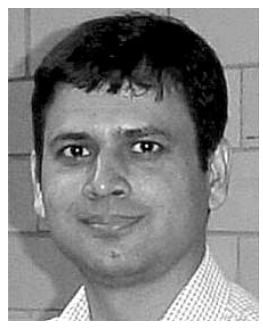

Kamran Akhtar (M'01) received the M.S. degree in physics from IIT Roorkee, India, the M.S degree in material sciences from IIT Kanpur, India, and the $\mathrm{Ph} . \mathrm{D}$. degree in plasma physics from IIT Delhi, India, in 1997.

From 1996 to 2001, he was with the Faculty of Engineering and Technology, Jamia Millia Islamia (a Central University by the act of Parliament), Delhi, India. Since 2001, he has been with the Department of Electrical and Computer Engineering at University of Wisconsin-Madison. His research interests include the production and characterization of high/moderate-density, large-volume microwave, radio-frequency, and laser produced laboratory plasmas for industrial and biological applications.

Dr. Akhtar was awarded the INFN Fellowship in 2001 by the government of Italy.

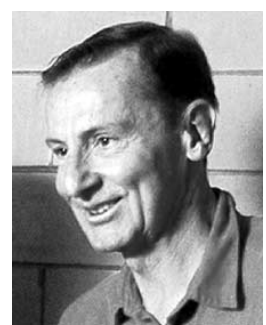

John E. Scharer (SM'90) received the B.S., M.S., and $\mathrm{Ph} . \mathrm{D}$. degrees in Electrical Engineering from the University of California Berkeley in plasma physics.

$\mathrm{He}$ is a professor in the Department of Electrical and Computer Engineering and Co-Director of the Center for Plasma Theory and Computation at University of Wisconsin-Madison. He has spent sabbaticals at the CEA Fontenary-aux-Roses, France, in 1970, at the JET tokamak at Culham, U.K., in 1983, and at the Australian National University, Sydney, Australia, in 2000, working on RF plasma physics. He has over 35 years of cumulative research experience in excimer laser plasma creation, optical spectroscopy, radiofrequency sustainment, millimeter-wave diagnostics and chemistry of organic seed gas plasmas in air and microwave vacuum electronics. He also has extensive experience in theoretical, computational and experimental research on antenna coupling and wave propagation, heating and creative diagnostics in plasmas and vacuum electronics.

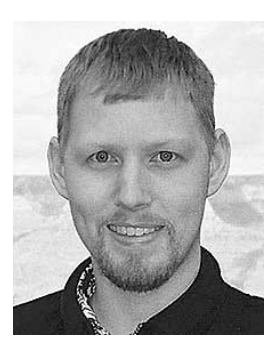

Shane Matthew Tysk originally from St. Paul, MN, received the B.S. degree in physics and the B.A. degree in applied mathematics from the University of St. Thomas, St. Paul, MN, in 2001, and the M.S degree in electrical engineering from the University of Wisconsin-Madison in 2003.

His research activities have included laser initiated high pressure atmospheric gas plasmas, helicon plasmas, laser cooling, and atom trapping.

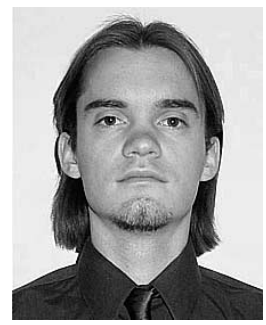

C. Mark Denning (M'01) received the B.S. degree in electrical engineering at the University of IllinoisChicago in 2002.

$\mathrm{He}$ is currently a research assistant in the Department of Electrical and Computer Engineering at the University of Wisconsin-Madison. His research interests include experimentation and computational modeling of helicon and high-pressure atmospheric gas plasmas. 